Notre Dame Journal of Formal Logic

Volume 42, Number 2, 2001

\title{
Pseudo Treealgebras
}

\author{
M. Bekkali
}

\begin{abstract}
A pseudotree $\langle T, \leq\rangle$ is a partially ordered set for which $\{u \in T$ : $u \leq t\}$ is a linear ordering for each $t \in T$. Define $\mathscr{B}(T)$, the pseudo treealgebra over $T$, as the subalgebra of the power set of $T$ generated by $\left\{b_{t}: t \in T\right\}$ where $b_{t}=\{u \in T: t \leq u\}$. It is shown that every pseudo treealgebra is embeddable into an interval algebra; thus it is a retractive Boolean algebra. Moreover, superatomicity of $\mathscr{B}(T)$ is described using conditions on $\langle T, \leq\rangle$.
\end{abstract}

\section{Elementary Material}

A pseudotree $T$ is a poset in which the set of predecessors of any element is a linearly ordered set. For $t \in T$, put $b_{t}=\{u \in T: t \leq u\}$. The subalgebra of the power set of $T$ generated by $\left\langle b_{t}: t \in T\right\rangle$ is called the pseudo treealgebra generated by $T$. Almost all properties of treealgebras remain valid in the case of pseudo treealgebras (see Brenner and Monk [1], Koppelberg [2], and Koppelberg and Monk [3]). Thus we can write a nonzero element of $\mathcal{B}(T)$ in its normal form (see [1]) and for a pseudotree with a least element, the Stone space $\operatorname{Ult}(\mathcal{B}(T))$ of a pseudo treealgebra $\mathcal{B}(T)$ is homeomorphic to $I_{c}(T)=$ the set of all initial chains endowed with Tychonoff's topology inherited from the catersian product ${ }^{T} 2$.

Throughout this note each pseudotree is assumed to have a single root as is shown by the following proposition.

Proposition 1.1 Any pseudo treealgebra is isomorphic to a pseudo treealgebra over a pseudotree with a single root.

Proof Let $\mathcal{B}(T)$ be a pseudo treealgebra.

Case $1 T$ has finitely many roots $t_{1}, \ldots, t_{n}$ and no rootless elements. Define $s \leq^{*} t$ if and only if $\left(s \leq_{T} t\right.$ or $\left(s=t_{1}\right.$ and $\left.\left.t \neq t_{1}\right)\right)$. Let $T^{*}$ be $T$ under $\leq^{*}$. Note that $\left\langle b_{t}^{T^{*}}: t \neq t_{1}\right\rangle=\mathscr{B}\left(T^{*}\right)$. Define $f\left(b_{t}^{T^{*}}\right)=b_{t}^{T}$ for all $t \neq t_{1}$. Then $f$ extends to an

Received July 9, 1998; accepted May 18, 2001; printed May 22, 2003

2001 Mathematics Subject Classification: Primary, 03G, 06A; Secondary, 06A05, 06B10, 06E05

Keywords: pseudo treealgebra, interval algebra, ordered set (c) 2003 University of Notre Dame 
isomorphism of $\mathscr{B}\left(T^{*}\right)$ into $\mathscr{B}(T)$ by Sikorski's Criterion (see Theorem 5.5, p. 67 in [2]).

Case $2 T$ has infinitely many roots or has a rootless element. Let $x \notin T$ and put $T^{*}=T \cup\{x\}$. Define $\leq^{*}$ on $T^{*}$ as follows:

$$
s \leq^{*} t \text { iff }\left(s, t \in T \text { and } s \leq_{T} t\right) \text { or }(s=x \text { and } t \in T) .
$$

Now put $f\left(b_{t}^{T^{*}}\right)=b_{t}^{T}$ for all $t \neq x$. Then $f$ extends, again, to an isomorphism of $\mathscr{B}\left(T^{*}\right)$ onto $\mathscr{B}(T)$ by Sikorski's Criterion.

Notice that chains are pseudotrees. Hence if $C$ is a chain, $\mathscr{B}(C)$ is called the interval algebra over $C$. The Stone space $\operatorname{Ult}(\mathscr{B}(C))$ is homeomorphic to the set of initial chains of $C$, denoted by $I(C)$, whenever $C$ has a least element. Superatomic interval algebras are characterized by the following theorem.

Theorem 1.2 The following are equivalent for any chain $C$ with a least element.

1. $\eta$, the chain of rational numbers with its natural ordering, does not embed into $C$;

2. $\eta$ does not embed into $I(C)$;

3. $(I(C), C)$ is a scattered topological space;

4. $\mathscr{B}(C)$ is a superatomic interval algebra.

First, we give a definition.

Definition 1.3 Let $X$ be a topological space. We say that $a \in A \subset X$ is an isolated point in $A$ whenever there exists an open set $U$, in $X$, containing $a$ so that $U \cap A=\{a\}$. Isol $(A)$ shall denote the set of isolated points of $A$ in $X$. Also, $\bar{A}$ denotes the topological closure of $A$ in $X$. A topological space $X$ is a scattered space whenever Isol $(F)$ is not empty for every nonempty closed subspace $F$ of $X$. Finally, a poset $(P,<)$ is scattered whenever the chain of rational numbers, under its natural ordering, does not embed in $(P,<)$.

Lemma 1.4 Let $C$ be a complete chain. If $C$ is a scattered topological space, then $\eta$ does not embed into $(C,<)$.

Proof First we note the following:

1. If $S \subseteq C$ is infinite, then $\bar{S} \backslash \operatorname{isol}(\bar{S}) \neq \varnothing$. This follows since $C$ is a compact. Now suppose that $S$ is a chain in $C$ of type $\eta$; we shall get a contradiction. Choose $x \in S^{\prime}={ }_{\operatorname{def}} \bar{S} \backslash \operatorname{isol}(\bar{S}), x$ isolated in $C$. Say, $u<x<v ;(u, v) \cap C=\{x\}$.

2. There are $s, t \in S$ so that $u<s<t<v$, and $x \notin[s, t]$. In fact, since $x \notin \operatorname{isol}(\bar{S})$, the set $(u, s) \cap \bar{S}$ is infinite. Hence there clearly exist $u<w_{1}<w_{2}<w_{3}<v$ such that $\left(w_{1}, w_{2}\right) \neq \varnothing \neq\left(w_{2}, w_{3}\right)$ and $x \notin\left(w_{1}, w_{2}\right)$. Choose $s \in\left(w_{1}, w_{2}\right) \cap S, t \in\left(w_{2}, w_{3}\right) \cap S$; this proves (2).

Taking $s$ and $t$ as in (2), put $S^{\prime}=(x, t) \cap S$. So $S^{\prime}$ has type $\eta$. Clearly $\overline{S^{\prime}} \backslash \operatorname{isol}\left(\bar{S}^{\prime}\right) \subseteq C$. Picking $w$ in $\bar{S}^{\prime} \backslash \operatorname{isol}\left(\bar{S}^{\prime}\right)$ by (1), we obtain $w \in(u, v) \cap C \backslash\{x\}$, contradiction.

Remark 1.5 The hypothesis that $C$ is complete in Lemma 1.4 is really needed. This is seen by the example $\omega \cdot \eta$ which is a scattered space. 
Proof of Theorem $1.2 \quad$ (3) and (4) are equivalent by the duality theory. (2) implies (1) since $C$ embeds in $I(C)$. (1) implies (4) since a quotient of $\mathcal{B}(C)$ is isomorphic to $\mathscr{B}\left(C^{\prime}\right)$ for some subchain $C^{\prime}$ of $C$ (see Theorem 15.22, p. 253 in [2]). Finally, (3) implies (2) by Lemma 1.4.

\section{Retractiveness of Pseudo Treealgebras}

Our approach to proving that every pseudo treealgebra is in fact a subalgebra of an interval algebra, and hence is a retractive algebra by Rubin's Theorem (see Theorem 15.22 , p. 253 in [2]), is done in a very canonical and constructive way compared to Theorem 16.12, p. 262 in [2]. In this fashion one will have a link between superatomicity of a pseudo treealgebra $\mathscr{B}(T)$ and the superatomicity of the canonical interval algebra in which $\mathscr{B}(T)$ embeds.

Let $T$ be a pseudotree. For each initial chain $p$ of $T$ set

$$
T_{p}={ }_{\text {def }}\left\{t \in T: s<_{T} t \text { for all } s \in p\right\} .
$$

Next we define $\equiv_{p}$ on $T_{p}$ by the following rule:

$$
t \equiv_{p} t^{\prime} \text { iff there is } s \in T \backslash p \text { such that } s \leq_{T} t, t^{\prime} .
$$

Note then that $s \in T_{p}$; for if $u \in p$, then $u \leq t, t^{\prime}$. So $u$ and $s$ are comparable, and $s \leq u$ is ruled out. So $u<s$. Thus $s \in T_{p}$.

Lemma 2.1 $\equiv_{p}$ is an equivalence relation on $T_{p}$.

Proof Suppose $t \equiv_{p} t^{\prime} \equiv_{p} t^{\prime \prime}$. Say $s, s^{\prime} \in T \backslash p$ and $s \leq t, t^{\prime}$ and $s^{\prime} \leq t^{\prime}, t^{\prime \prime}$. So $s, s^{\prime}$ are comparable. Say $s \leq s^{\prime}$. Thus $s \leq t, t^{\prime \prime}$ and so $t \equiv_{p} t^{\prime \prime}$.

Next, put $s \wedge t==_{\operatorname{def}}\{u \in T: u<s, \bar{t}\}$ and fix a well-ordering $\preceq_{p}$ on $T_{p} / \equiv_{p}$. Define $\leq \operatorname{lin}$ on $T$ as follows:

$$
s \leq_{\text {lin }} t \text { iff }\left\{\begin{array}{l}
s \leq t \text { in } T, \text { or } \\
s, t \text { are incomparable in } \mathrm{T} \text { and }[s]_{\equiv_{s \wedge t}} \preceq_{s \wedge t}[t]_{\equiv_{s \wedge t}}
\end{array}\right.
$$

where $[s]_{\equiv_{s \wedge t}},[t]_{\equiv_{s \wedge t}}$ denote the equivalence classes of $s, t$ with respect to $\equiv_{s \wedge t}$.

Lemma 2.2 $\leq$ lin is a linear ordering on $T$.

Proof Clearly $\leq_{\text {lin }}$ is irreflexive and for all $s, t$ in $T, s \leq_{\operatorname{lin}} t$ or $t \leq \operatorname{lin} s$. Now suppose $x \leq \operatorname{lin} y \leq \operatorname{lin} z$.

Case $1 x<y<z$. So $x<z$. Thus $x<\operatorname{lin} z$.

Case $2 x<y ; y, z$ are incomparable in $T,[y]_{\equiv_{y \wedge z}}<_{y \wedge z}[z]_{\equiv_{y \wedge z}}$. If $x<z$, we are done. Thus, assume $x \not z z$. Clearly $z \not \leq x$. We claim now that $x \wedge z=y \wedge z$. Clearly $x \wedge z \subseteq y \wedge z$. Suppose $w \in y \wedge z$. Thus $w<y$, so $w, x$ are comparable. If $x \leq w$, then $x<z$, contradiction. So $w<x$. Thus $x \wedge z=y \wedge z$. Clearly $[x]_{\equiv_{y \wedge z}}=[y]_{\equiv_{y \wedge z}}$. So $x<\operatorname{lin} z$.

Case $3 x, y$ incomparable in $T .[x]_{\equiv_{x \wedge y}}<_{x \wedge y}[y]_{\equiv_{x \wedge y}} ; y<z$. This case is similar to Case 2.

Case $4 x, y$ incomparable in $T .[x]_{\equiv_{x \wedge y}}<_{x \wedge y}[y]_{\equiv_{x \wedge y}} ; y, z$ are incomparable in $T$, $[y]_{\equiv_{y \wedge z}}<y \wedge z[z]_{\equiv_{y \wedge z}}$.

Subcase 4.1 $x \wedge y=y \wedge z$.

1. $x<z$. Thus $x<\operatorname{lin} z$. 
2. $x \equiv_{x \wedge y} z$. For $z \leq z, z<x$, and $z \notin x \wedge y(=y \wedge z)$. So $[x]_{\equiv_{x \wedge y}}=[z]_{\equiv_{x \wedge y}}$. Therefore, by the assumption in this case $[x]_{\equiv_{x \wedge y}}=[z]_{\equiv_{x \wedge y}} \preceq_{x \wedge y}[y]_{\equiv_{x \wedge y}}$ $\preceq_{x \wedge y}[z]_{\equiv_{x \wedge y}}$ since $x \wedge y=y \wedge z$. Hence, $[z]_{\equiv_{x \wedge y}} \preceq_{x \wedge y}[z]_{\equiv_{x \wedge y}}$, contradiction.

3. $x, z$ incomparable in $T$. Since $x \wedge y=y \wedge z$, we have $[x]_{\equiv_{x \wedge y}} \preceq_{x \wedge y}[y]_{\equiv_{x \wedge y}}$ $\preceq_{x \wedge y}[z]_{\equiv_{x \wedge y}}$. Now $x \wedge y=x \wedge z$. For $x \wedge y \subseteq x \wedge z$ is clear, and if $w \in x \wedge z \backslash x \wedge y$, then $[x]_{\equiv_{x \wedge y}}=[z]_{\equiv_{x \wedge y}}$, contradiction. So $[x]_{\equiv_{x \wedge z}} \preceq_{x \wedge z}[z]_{\equiv_{x \wedge z}}$ follows.

Subcase $4.2 x \wedge y \neq y \wedge z$.

1. There is $w \in x \wedge y \backslash y \wedge z$. Thus $w<x, x<y$, and $w \not z z$.

2. $x, z$ are incomparable. In fact $x \leq z z$. Otherwise $w<z$, and if $z<x$, then $w, z$ are comparable. Hence $z \leq w<y$, contradiction.

3. $x \wedge z=y \wedge z$. Let $t \in x \wedge z$. Then $t<x$, so $t, w$ are comparable. If $w \leq t$, then $w<z$, contradiction. So $t<w$. Hence $w<z$, contradiction. So $t \leq w$, hence $t<x$ as desired.

Theorem 2.3 Any pseudo treealgebra embeds into an interval algebra and thus it is a retractive Boolean algebra.

Proof Let $\mathcal{B}(T)$ be a pseudo treealgebra. First of all we may assume that $T$ has no maximal element. To this end, define $\breve{T}$ to be $T$, and add a well-ordered chain $C_{t}$ of type $\omega$ above each maximal element $t$ in $T$. Hence $\breve{T}$ has no maximal element and by copying the proof of Theorem 16.7, p. 260 in [2], $\mathscr{B}(T)$ embeds in $\mathscr{B}(\breve{T})$.

So suppose $T$ has no maximal element and denote by $L$ the completion of $\langle T, \leq \operatorname{lin}\rangle$. For each $t \in T$, let $y_{t}=\sup _{L}\left(b_{t}\right)$. Note that $y_{t} \in L \backslash T$. Let $0_{T}$ be the root of $T$ and define $f$ from $\mathscr{B}(T)$ into the interval algebra over $L \backslash\left\{y_{0_{T}}\right\}$ by

$$
f\left(b_{t}\right)=\left[t, y_{t}\right) .
$$

Notice that $f\left(b_{t}\right)=0$ if and only if $t=y_{t}$ if and only if $t$ is maximal in $T$; but this never happens.

Next $f$ extends to an isomorphism of $\mathscr{B}(T)$ into $\operatorname{Int}\left(L \backslash\left\{y_{0_{T}}\right\}\right)$. Indeed, look at

$$
b_{t(1)}, \ldots, b_{t(m)}-b_{s(1)}-\cdots-b_{s(n)} .
$$

If $(*)$ is zero, we get then three cases.

Case 1 There are $i, j$ so that $t(i), t(j)$ are incomparable. Then either every element of $b_{t(i)}$ is $\leq$ lin-less than every element of $b_{t(j)}$ or conversely. In any case we get

$$
f\left(b_{t(i)}\right) \cap f\left(b_{t(j)}\right)=\varnothing .
$$

Case 2 There are $i, j$ such that $s_{i} \leq t_{j}$. Thus $b_{t(j)} \subseteq b_{s(i)}$. So $y_{t(j)} \leq y_{s(i)}$, $f\left(b_{s(i)}\right) \supseteq f\left(b_{t(j)}\right)$ as desired.

Case 3 There is an $i_{0} \in[1, n]: s\left(i_{0}\right)=0_{T}$. So $f\left(b_{s\left(i_{0}\right)}\right)=f\left(1_{\mathcal{B}(T)}\right)=$ $\left[0_{T}, y_{0_{T}}\right)=L \backslash\left\{y_{0_{T}}\right\}=1$. Thus $f$ extends by Sikorski's Criterion to a homomorphism from $\mathscr{B}(T)$ into $\operatorname{Int}\left(L \backslash\left\{y_{0_{T}}\right\}\right)$. Suppose that $(*)$ is not zero. Without loss of generality $m \neq 0$. If $t(i)$ is maximal among $t(1), \ldots, t(m)$, clearly $t(i)$ is in the image of $(*)$. This finishes up the proof of Theorem 2.3. 


\section{Characterization of Superatamic Pseudo Treealgebras}

Theorem 3.1 Let $T$ be a pseudotree. The following statements are equivalent.

1. $\mathcal{B}(T)$ is a superatomic Boolean algebra.

2. $\eta$ and the binary tree ${ }^{<\omega} 2$ do not embed in $\langle T, \leq\rangle$.

The main step in proving this theorem is Lemma 3.4 below. So denote by $E$ the set $T \cup\left\{y_{t}: t \in T\right\} \backslash\left\{y_{0_{T}}\right\}$, where $T$ is a pseudotree without maximal elements, and recall that $y_{t}$ denotes $\sup \left(b_{t}\right)$ in the completion of $\left(T, \leq_{\text {lin }}\right)$. Notice that this assumption on $T$ does not restrict the generality as shown by the following two facts. Recall that $\breve{T}$ is constructed as in the beginning of the proof of Theorem 2.3.

Fact 3.2 For any pseudotree, the following statements are equivalent.

1. $\eta$ or $<\omega 2$ embeds into $\mathrm{T}$.

2. $\eta$ or $<\omega 2$ embeds into $\breve{T}$.

Fact 3.3 $\mathscr{B}(T)$ is superatomic if and only if $\mathcal{B}(\breve{T})$ is.

Lemma 3.4 The following statements are equivalent.

1. E contains $\eta$.

2. Either $\eta$ or ${ }^{<\omega} 2$ embeds in $T$.

Assuming Lemma 3.4 we give the proof of Theorem 3.1.

\section{Proof of Theorem 3.1}

$\neg(2)$ implies $\neg$ (1) $\quad$ If $\eta$ or $<\omega 2$ embeds in $T$, then $\operatorname{Int}(\eta)$ or $\mathscr{B}\left(T_{\omega}\right)$ embeds in $\mathscr{B}(T)$, where $T_{\omega}$ is the tree of height $\omega$ so that any node in $T_{\omega}$ has $\omega$ immediate successors. Hence (1) implies (2) follows.

$\neg(1)$ implies $\neg$ (2) If $\mathcal{B}(T)$ is not superatomic, then by Fact 3.3 neither is $\mathcal{B}(\breve{T})$. Forming $E$ as we stated previously, it follows that $\operatorname{Int}\left(E \backslash\left\{y_{0_{T}}\right\}\right)$ is not superatomic. So $\eta \leq E$. So by Lemma 3.4, $\eta$ or ${ }^{<\omega} 2$ embeds in $\breve{T}$. Hence by Fact $3.2 \eta$ or ${ }^{<\omega} 2$ embeds into T. This finishes up the proof of Theorem 3.1.

\section{Proof of Lemma 3.4}

(2) implies (1) If $\eta$ embeds in $\left\langle T, \leq_{T}\right\rangle$ then it embeds into $E$ by the above. Suppose that ${ }^{<\omega} 2$ embeds into $\left\langle T, \leq_{T}\right\rangle$. Then so does $T_{\omega}$, where $T_{\omega}$ is of height $\omega$, has one root, and each element has $\omega$ immediate successors. Hence $\mathcal{B}\left(T_{\omega}\right)$ (which is atomless) embeds into $\operatorname{Int}(E)$. Hence (1) follows.

(1) implies (2) Suppose that $\eta$ does not embed in $\langle T, \leq T\rangle$. Let $F$ be a subset of $E$ of type $\eta$. Because of the following fact, we may assume that $F \subseteq T$.

Fact 3.5 If a linear ordering $L$ is scattered, so is its completion.

Proof Since $L$ is scattered, so is $I(L)$ (by Theorem 1.2). Next, since the completion of $L$ is order embeddable in $I(L)$, it follows that the completion of $L$ is scattered as well.

Now back to the proof of Lemma 3.4. $F$ cannot be a chain in $T$ since $\eta \not \leq T$. Choose $u_{0}, v_{0} \in F$ such that $u_{0}, v_{0}$ are incomparable; say $u_{0}<$ lin $v_{0}$. Pick $w_{0} \in u_{0} \wedge v_{0}$. It 
suffices now to prove the following.

\section{There exist $u_{1}, u_{2}, v_{1}, v_{2}, w_{1}, w_{2}$ so that}

1. $u_{i}, v_{i} \in F$ for $i=1,2$,

2. $u_{i}$ and $v_{i}$ are incomparable for $i=1,2$,

3. $u_{i}<\operatorname{lin} v_{i}$ for $i=1,2$,

4. $w_{1}, w_{2}$ are upper bounds of $u_{0}, v_{0}$ in $(T,<)$, $w_{i}$ is in $u_{i} \wedge v_{i}$ for $i=1,2$ and $w_{1}, w_{2}$ are incomparable.

First set

$\left(u_{0}, v_{0}\right)_{T}=_{\operatorname{def}}\left\{u \in T: u_{0}<\operatorname{lin} u<\operatorname{lin} v_{0}\right\}$,

$\Omega=\left\{s \wedge t: s, t \in\left(u_{0}, v_{0}\right)_{T} \cap F ; s, t\right.$ are incomparable elements of $\left.\mathrm{T}\right\}$.

Second, $\Omega \neq \varnothing$ since $\left(u_{0}, v_{0}\right)_{T} \cap F$ cannot be a chain.

Lemma 3.6 $(\Omega, \supseteq)$ is not a chain.

Proof The proof of this lemma uses the following claims. Indeed, suppose the contrary, and let $\mathscr{D}$ be the union of all members of $\Omega$.

Claim 3.7 If $t, t^{\prime} \in T$ are incomparable, $s \in T$, and $t<\operatorname{lin} s<\operatorname{lin} t^{\prime}$; then $t \wedge t^{\prime}<s$, that is, for all $w \in t \wedge t^{\prime}(w<s)$.

Proof For if $t<s$, obviously $t \wedge t^{\prime}<s$. So assume $t, s$ are incomparable. If $s<t^{\prime}$, take any $w \in t \wedge t^{\prime}$. So $w, s$ are comparable. If $s \leq w$, then $s<t$, contradiction. So $w<s$. So $t \wedge t^{\prime}<s$. Hence we may assume $s, t^{\prime}$ are incomparable. Now all elements of $\left(t \wedge t^{\prime}\right) \cup(t \wedge s)$ are comparable since all are less than $t$. Hence $t \wedge s \subseteq t \wedge t^{\prime}$ or $t \wedge t^{\prime} \subseteq t \wedge s$. Suppose $t \wedge s \subset t \wedge t^{\prime}$. Pick $w \in\left(t \wedge t^{\prime}\right) \backslash(t \wedge s)$. So $w>s$. Thus $t \equiv_{t \wedge s} t^{\prime}$. We claim that $t \wedge s=t^{\prime} \wedge s$. One needs only show $t^{\prime} \wedge s \subseteq t \wedge s$ since the other inclusion is clear by supposition. Suppose $u \in t^{\prime} \wedge s$. Thus $w, u$ are comparable since both are less than $t^{\prime}$. If $w \leq u$, then $w \leq s$, contradiction. So $u<w$. Hence $u<t$. This proves our assertion. Now $[t]_{\equiv_{t \wedge s}} \preceq[s]_{\equiv_{t \wedge s}}$. So $\left[t^{\prime}\right]_{\equiv_{t^{\prime} \wedge s}} \preceq[s]_{\equiv_{t^{\prime} \wedge s}}$. Hence $t^{\prime}<\operatorname{lin} s$, contradiction. This shows that $t \wedge t^{\prime} \subseteq t \wedge s$, and Claim 3.7 holds.

For each $t \in T$, put $T \downarrow t=\left\{u \in T: u \leq_{T} t\right\}$ and for each $G \subseteq \mathscr{D}$ set

$$
T(G)=\left\{t \in\left(u_{0}, v_{0}\right)_{T} \cap(F \backslash \mathscr{D}):(T \downarrow t) \cap \mathscr{D}=G\right\} .
$$

Claim 3.8 If $t, t^{\prime}$ are members of $T(G)$ and are incomparable, then $t \wedge t^{\prime}=G$.

Proof Assume the hypothesis. Then $t \wedge t^{\prime} \in \Omega$, so $t \wedge t^{\prime} \subseteq \mathscr{D}$. If $u \in t \wedge t^{\prime}$, then $u$ is in $(T \downarrow t) \cap \mathscr{D}=G$; if $u \in G$, then $u \in(T \downarrow t) \cap\left(T \downarrow t^{\prime}\right)=t \wedge t^{\prime}$. So Claim 3.8 holds.

Claim 3.9 If $t \in T(G)$ and $a \in[t]_{\equiv_{G}} \cap\left(u_{0}, v_{0}\right)_{T} \cap(F \backslash \mathcal{D})$, then $a \in T(G)$.

Proof Say that $G<x$ (i.e., $x$ is above all members of $G$ ) and $x \leq a, x \leq t$. Since $(T \downarrow t) \cap \mathscr{D}=G$, we have $x \notin \mathscr{D}$. Hence $(T \downarrow a) \cap \mathscr{D}=G$. So $a \in T(G)$.

Claim 3.10 If $t \in T(G)$, then $[t]_{\equiv_{G}} \cap\left(u_{0}, v_{0}\right)_{T} \cap(F \backslash D)$ is a chain in $T$.

Proof Let $a, b \in[t]_{\equiv_{G}} \cap\left(u_{0}, v_{0}\right)_{T} \cap(F \backslash \mathcal{D})$, and suppose that they are incomparable. Claim 3.8 and Claim 3.9 hold. By Claim 3.9, $a, b \in T(G)$ and so by Claim 3.8 $a \wedge b=G$, contradicting $a \equiv_{G} b$. 
Claim 3.11 If $t \in T(G)$, then $[t]_{\equiv_{G}} \cap\left(u_{0}, v_{0}\right)_{T} \cap(F \backslash \mathscr{D})=\{t\}$.

Proof Suppose the left-hand side has more than two elements. By Claim 3.10 and $\eta \not \leq T$, let $a<b$ be in the left-hand side, and no member of the left-hand side between them. Say $a<\operatorname{lin} c<\operatorname{lin} b, c \in F$. Suppose $a, c$ are incomparable in $(T,<)$. Now $a \wedge c=b \wedge c$. For $a \wedge c \subseteq b \wedge c$ is clear. Suppose $x \in b \wedge c$. Now $a<b, x<b$, so $x, a$ are comparable. Note that $b, c$ are incomparable ( $c<b$ implies $a, c$ are comparable, which is a contradiction). So $b \wedge c \in \Omega, b \wedge c \subseteq \mathscr{D}$. If $a<x$, then $a \in \mathscr{D}$, contradiction. So $x<a$. Thus $a \wedge c=b \wedge c .[a]_{\equiv_{a \wedge c}}<[c]_{\equiv_{a \wedge c}}, a \equiv_{a \wedge c} b$. So $[b]_{\equiv_{b \wedge c}}<[c]_{\equiv_{b \wedge c}}, b<\operatorname{lin} c$, contradiction. It follows that $a<c$. Hence $c \in[t]_{\equiv_{G}} \cap\left(u_{0}, v_{0}\right)_{T} \cap(F \backslash \mathcal{D})$, so by Claim 3.10, $b$ and $c$ are comparable, hence $c<b$, contradicting the choice of $a$ and $b$.

Claim 3.12 If $u \in T(G), t \in\left(u_{0}, v_{0}\right)_{T} \cap F$, and $t<u$, then $t \in \mathscr{D}$.

Proof For otherwise Claim 3.11 is contradicted.

An element $t \in\left(u_{0}, v_{0}\right)_{T} \cap(F \backslash \mathscr{D})$ is left of $\mathscr{D}$ whenever it is less than $u$ in $(E,<\operatorname{lin})$ for some $u \in \mathscr{D}$. Suppose there exist such $t, u$. For $G \subseteq \mathscr{D}$ let $T^{\prime}(G)$ be defined by

$$
T^{\prime}(G)=\{s \in T(G): s<\operatorname{lin} t\} .
$$

Suppose $\left|T^{\prime}(G)\right| \geq 2$ for some $G$. By Claim 3.8 through Claim 3.11, $\left(T^{\prime}(G),<\operatorname{lin}\right)$ cannot be in itself and thus choose $s<\operatorname{lin} s^{\prime}$ both in $T^{\prime}(G)$, with no member of $T^{\prime}(G)$ between them. Choose $v \in F$ such that $s<\operatorname{lin} v<\operatorname{lin} s^{\prime}$. Note that $s, s^{\prime}$ are incomparable by Claim 3.11 and hence by Claim 3.7, $s \wedge s^{\prime}<v$. If $b \in T(G)$, then since $v<\operatorname{lin} s^{\prime}<\operatorname{lin} t, v \in T^{\prime}(G)$, contradicting the choice of $s, s^{\prime}$. So there is an $x \in \mathscr{D}$, with $G<x \leq v$. Since $s<\operatorname{lin} t<\operatorname{lin} u$, we have $u \notin G$. So $s \wedge s^{\prime}=s \wedge u=s^{\prime} \wedge u=G$. Also, $s^{\prime} \wedge v=G$. In fact, $s^{\prime} \wedge v \supseteq G$ is true since $s \wedge s^{\prime}<v$, and to see that $s^{\prime} \wedge v \subseteq G$, assume that $r<s^{\prime}, r<v$. So $r$ and $x$ are comparable. If $r \leq x$, then $r \in \mathscr{D}$ and hence $r \in G$, as desired. If $x<r$, then $x<s^{\prime}$, hence $x \in G$, contradiction. Now $[s]_{\equiv_{G}}<\left[s^{\prime}\right]_{\equiv_{G}}<[u]_{\equiv_{G}}=[v]_{\equiv_{G}}$, so $s^{\prime}<\operatorname{lin} v$, contradiction. So $\left|T^{\prime}(G)\right| \leq 1$, for all $G$. Let

$$
\Omega^{\prime}=\left\{s \wedge s^{\prime}: s, s^{\prime} \text { are incomparable members of }\left(u_{0}, t\right)_{T} \cap F\right\} .
$$

Notice that $\Omega^{\prime} \subset \Omega$ and by our assumption $(\Omega, \supset)$ is assumed to be a chain. Thus $\Omega^{\prime} \neq \varnothing$. Now $\left|\Omega^{\prime}\right| \geq 2$. Suppose $\Omega^{\prime}=\{G\}$. Pick incomparable elements $s, s^{\prime}$ in $\left(u_{0}, t\right)_{T} \cap F$. So $s \wedge s^{\prime}=G$. Say $s \notin \mathcal{D}$. Pick incomparable $w, w^{\prime} \in\left(u_{0}, s\right)_{T} \cap F$. Say $w \notin \mathscr{D}$. Then $w \in T^{\prime}(G), s \in T^{\prime}(G), w \neq s$, but this contradicts $\left|T^{\prime}(G)\right| \leq 1$. So $\left|\Omega^{\prime}\right| \geq 2$.

The next fact follows easily.

Fact 3.13 If $D$ is a chain and $E=I(D)$ is the set of all initial segments of $D$, then $D$ is scattered if and only if $(E, \supseteq)$ is scattered.

Hence, notice that $(\Omega, \supset)$ is scattered since $\eta \not \leq(T,<)$ and thus choose $G, H \in \Omega^{\prime}$ with $G \subseteq H$, so that no member of $\Omega^{\prime}$ is between them. Pick $s \in T(G), s^{\prime} \in T(H)$. So $s \in T^{\prime}(G), s^{\prime} \in T^{\prime}(H)$. Note that $s \wedge s^{\prime}=G=s \wedge u$, so any $h \in H \backslash G$ shows that $s^{\prime} \equiv_{G} u$ and so $s<\operatorname{lin} s^{\prime}$. Pick incomparable $w, w^{\prime}$ in $\left(s, s^{\prime}\right)_{T} \cap F$. Say $w \notin D D$. Say $w \in T(K)$. So $w \in T^{\prime}(K)$. Now $s$ and $s^{\prime}$ are incomparable by Claim 3.11. So $s \wedge s^{\prime}<w$ by Claim 3.7, that is, $G<w$. Hence $H \subset K$ by the choice of $G$ and $H$ plus $T^{\prime}(G)=\{s\}, T^{\prime}(H)=\left\{s^{\prime}\right\}$. But then $s^{\prime}<\operatorname{lin} u$ implies 
$s^{\prime}<\operatorname{lin} w$ (by considering an element of $K \backslash H$ ), contradiction. Thus, no element of $\left(u_{0}, v_{0}\right)_{T} \cap(F \backslash \mathcal{D})$ is left of $\mathscr{D}$. Suppose $|T(G)| \geq 2$, for some $G$. Let $t<\operatorname{lin} t^{\prime}$ both in $T(G)$ with no element of $T(G)$ between them. We easily reach a contradiction as in the case $\left|T^{\prime}(G)\right| \geq 2$ above. So $|T(G)| \leq 1$ for all $G$. Then we reach a contradiction as above. This finishes up the proof of Lemma 3.6.

So $(\theta)$ is finally established. Choose $G, H \in \Omega$, incomparable. Take $w_{1} \in G \backslash H$, $w_{2} \in H \backslash G$. Without loss of generality, $w_{1}<\operatorname{lin} w_{2}$. Clearly, $w_{1}, w_{2}$ are incomparable. Say $u_{1}<\operatorname{lin} v_{1}, u_{1}$ and $v_{1}$ are incomparable, $u_{1}, v_{1} \in F \cap\left(u_{0}, v_{0}\right)_{T}$, and $u_{1} \wedge v_{1}=G$. Similarly, we get $u_{2}, v_{2}$ in $H . u_{0}<\operatorname{lin} w_{1}<\operatorname{lin} v_{0}$; so $u_{0} \wedge v_{0}<w_{1}$ by Claim 3.7. Hence we are through with the proof of Lemma 3.4.

\section{References}

[1] Brenner, G., and D. Monk, "Tree algebras and chains," pp. 54-66 in Universal Algebra and Lattice Theory, edited by R. S. Freese and O. C. García, vol. 1004 of Lecture Notes in Mathematics, Springer, Berlin, 1983. Zbl 0527.06007. MR 84m:06022. 101

[2] Koppelberg, S., Handbook of Boolean Algebras. Vol. 1, edited by J. D. Monk and R. Bonnet, North-Holland Publishing Co., Amsterdam, 1989. Zbl 0671.06001. MR 90k:06002. 101, 102, 103, 104

[3] Koppelberg, S., and J. D. Monk, "Pseudo-trees and Boolean algebras," Order, vol. 8 (1991/92), pp. 359-74. Zbl 0778.06011. MR 93i:06006.

\section{Acknowledgments}

We thank Professors D. Monk and S. Todorcevic for all the fruitful discussions we had during the preparation of this article and the referee for his suggestions. This work was supported by the Universtity of Colorado at Boulder, USA. This paper is dedicated to Fatima Al Fihria who founded the first university in Morocco in the eleventh century.

Department of Mathematics

MBA University Fez

MOROCCO

bekka@menara.ma 